\title{
NOUVEAUX ASPECTS DE LA THÉRAPIE LACTIQUE
}

\author{
par
}

\author{
JEAN PIEN
}

Ingénieur Chimiste (I. C. R.). Docteur ès Sciences

Directeur des Laboratoires de la laiterie des " Fermiers Réunis ".

\section{PREMIËRE PARTIE}

\section{RÉSUMÉ DE NOS CONNAISSANCES ACTUELLES CONCERNANT L'ÉTIOLOGIE DES COLIBACILLOSES ET DES ÉTATS TOXIQUES}

On réunit sous le terme d'ailleurs un peu impropre de colibacilloses un grand nombre de troubles complexes et multiformes, ayant leur origine dans un état chimique et bactériologique anormal $\mathrm{du}$ contenu intestinal.

Ces troubles, dont les manifestations cliniques des plus variées sont parfois déroutantes et dont le diagnostic exact est souvent difficile, se traduisent tantôt par des altérations du fonctionnement digestif ("embarras gastriques ) fébriles, constipation, diarrhée, mauvaise haleine, langue chargée), tantôt par des réactions nerveuses pénibles (migraines, anxiété, mélancolie, découragement, irratibilité, fatigue sans cause apparente), ou encore par des signes localisés par exemple à l'épiderme (formes d'urticaire, dermatoses), au système vasculaire (phlébites), à l'arbre urinaire (néphrites, uréthrites, cystites), au foie et à la vésicule (insuffisance hépatique, cholécystite non calculeuse) voire même au poumon, au tissu osseux, etc.

Il est vrai qu'un certain nombre de ces symptômes apparaissent à l'occasion d'états pathologiques n'ayant rien de commun avec les intoxications d'origine intestinale. Mais il est parfaitement reconnu que la sanction certaine de ces intoxications, de ces "colibacilloses " est l'apparition de troubles isolés ou multiples dont nous venons de rappeler quelques-unes des manifestations essentielles.

C'est donc le plus souvent au niveau de l'intestin qu'il convient de situer la eause de ces états toxiques.

Dégageons brièvement la genèse de ces formes variées de l'autointoxication et essayons de montrer les mécanismes qui les relient à leurs causes.

$$
* *
$$

\section{Evolution de la flore intestinale.}

Les microorganismes de l'intestin sont-ils indispensables à la vie?

Certains animaux, rares il est vrai, présentent une absolue stérilité de l'intestin. Il en est ainsi de quelques formes d'insectes métaboles, tout au moins dans leur phase larvaire (ver à soie, 
par exemple). Dans ce cas, il est vrai, la forme imago ou adulte comporte un intestin septique. Mais il est remarquable de constater que chez les larves actives, se nourrissant de végétaux, absorbant. incontestablement des microorganismes, la stérilité de l'intestin est. de règle et ce fait n'est nullement incompatible avec la vie.

Certains autres animaux, même à l'état adulte, ont également. un contenu intestinal presque aseptique.

Chez les mammifères et chez l'homme en particulier, le jeune, à sa naissance, possède une flore intestinale d'ane remarquable pauvreté qualitative. Les germes y sont nombreux mais représentés. presque exclusivement par une seule espèce, un bacille lactique particulier qui ne pent pas et ne doit pas être confondu avec les ferments lactiques ordinaires auxquels nous sommes habitués.

Ce lactobacille découvert par Mono il y a une quarantained'années présente plusieurs formes extrêmement voisines dont le chef de groupe est le Lactobacillus acidophilus.

Cette flore, qui communique aux selles une réaction nettement acide, conserve son caractère primitif pendant un temps variable chez le nourrisson et évolue ensuite vers une composition profondément différente.

Chez l'enfant et plus encore chez l'adulte, les fèces deviennent presque neutres, les bacilles lactiques naturels disparaissent à peu près complètement et sont remplacés par de nombreux autres microorganismes (entérocoque, colibacille, streptocoques et staphylocoques variés, bactéries de la putréfaction, etc.).

Or, sous des influences diverses liées à la composition des régimes alimentaires, à l'irrégularité du fonctionnement intestinal, à certaines affections, au genre de vie, etc,, on assiste à une exaltation de la prolifération, voire de la virulence de certains de ces hôtes de l'intestin, accompagnée le plus souvent de modifications de la réaction des fèces et même d'un fléchissement de l'intégrité de la muqueuse intestinale.

C'est cet ensemble de phénomènes complexes, où l'évolution de la flore intestinale tient la plus large place, qui est à l'origine des troubles plus ou moins graves rassemblés sous le terme général d'auto-intoxication ou de colibacillose.

Par quels mécanismes cés causes entraînent-elles les troubles que nous avons énumérés?

$$
*^{*} *
$$

\section{Mécanisme de l'auto-intoxication.}

La part actuellement connue des mécanismes qui sont à l'origine de ces intoxications, peut s'exprimer schématiquement de la manière suivante : 
$1^{0}$ De nombreux microorganismes intestinaux et en particulier les germes de la putréfaction produisent des substances toxiques : corps a fonction phénoliques, indol, etc. Le colibacille lui-même dont on a parfois tendance à sous-estimer les inconvénients directs, produit deux sortes de toxines : une endotoxine entérotrope, dont l'action irritante se manifeste sur l'intestin et se traduit par la diarrhée, et une exotoxine neurotrope qui se fixe sur les centres nerveux pour lesquels elle possède une affinité toute spéciale (travaux de VINCENT).

$2^{\circ}$ Un grand nombre des aminoacides libérés par le travail digestif peuvent subir des transformations anormales et dangereuses : par perte de la partie carboxylée de leur molécule, ils libèrent des amines dont certaines sont très toxiques, alors que le cycle normal devrait les conduire à la perte de leur partie azotée avec formation de substances non toxiques.

Cette transformation indésirable des aminoacides se produit généralement à l'occasion d'une variation du $p H$ du contenu intestinal.

Or, ces toxines microbiennes et ces substances chimiques toxiques sont résorbées par la muqueuse intestinale et passent dans le sang avec une plus ou moins grande intensité suivant l'état physiologique de cette paroi.

Il arrive même, et très fréquemment, que certains microorganismes $d u$ contenu intestinal (colibacille, entérocoque, staphylocoques et streptocoques) traversent eux-mêmes cette paroi à la faveur d'une défaillance tissulaire.

Les lésions microscopiques de la muqueuse intestinale, grâce à quoi peut avoir lieu le passage des microbes dans le sang, se produisent sous l'influence de causes variées : entérite, embarras gastrointestinal, action des parasites intestinaux (vers, amibes), usage de laxatifs, de lavements qui irritent et lèsent la muqueuse, etc.

Ces causes de décapage ou d'altération de la paroi de l'intestin se produisent très fréquemment. On a pu dire que cette inflammation de l'intestin, produite ou entretenue par des causes si diverses, constituait le terrain habituel de la colibacillose.

D'ailleurs sur la réalité du passage des microbes au travers des brèches accidentelles de la muqueuse il n'y a aucun doute possible, puisqu'aussi bien c'est sur ce principe qu'est basée la vaccination par voie buccale (BESREDKA) où l'ingestion des vaccins est précédée ou accompagnée d'une irritation légère susceptible de provoquer la fixation et la traversée des corps microbiens du milieu intestinal au milieu intérieur proprement dit.

Les microorganismes de l'intestin ayant pu, à la faveur de ces fines lésions, pénétrer dans la circulation vont traverser certains organes ou s'y fixer, ou même se répandre dans toute l'économie. 
Tous les organes, tous les tissus peuvent ainsi être atteints plus ou moins profondément par des germes qui n'auraient jamais dû franchir la barrière intestinale ou même simplement par leurs toxines dont la sortie est encore plus facile. C'est ainsi que l'on peut retrouver des localisations de ces microorganismes dans les reins et les voies urinaires, les organes génitaux, le foie, la vésicule biliaire, le poumon, les gencives, les veines, le système nerveux, les os, les articulations, etc.

C'est ce qu'exprime GAEHLINGER quand il écrit : "Lorsque ces microorganismes sont passés dans la circulation avant d'être rejetés dans les voies urinaires, ils peuvent donner lieu à des accidents toxiques ou infectieux au niveau des autres organes de l'économie, traduisant ainsi la dissémination des microbes et de leurs poisons, "

Signalons quelques-unes de ces localisations les plus importantes :

Les voies urinaires (syndrome entéro-rénal de HEITz-Boyter). Le colibacille est plus spécialement l'agent de cette implantation fréquente dont les conséquences sont nombreuses : néphrites, cystiques, prostatites, etc. C'est peut-être la forme la plus connue, la plus facile à déceler et souvent aussi la plus facile à guérir des colibacilloses. Les autres manifestations sont généralement plus difficiles à traiter et plus graves.

Le foie et la vésicule. Le colibacille et l'entérocoque peuvent déterminer des troubles hépatiques variés. Parfois l'infection se localise à la vésicule (cholécystite chronique non calculeuse). C'est le syndrome entéro-hépatique de DEsGeorges, dont le point de départ est bien l'infection intestinale avec localisation au système biliaire.

Certes, les poisons d'origine chimique (phénols, scatol, amines, etc.), issus de l'intestin et passant dans le foie en l'irritant, suffiraient à expliquer la plupart des troubles hépatiques d'origine intestinale. "C'est la vieille théorie de l'auto-intoxication. Elle a été complétée et rajeunie par cette notionn ouvelle que non seulement leurs produits mais encore les germes eux-mêmes peuvent dépasser la barrière de la muqueuse et atteindre le foie et les voies biliaires. " (GOIFFON.) foie?

Que résulte-t-il de cette intoxication, dans le travail normal du

Sous l'influence de ces poisons d'origine chimique ou microbienne, nous assistons à une surcharge de la fonction d'arrêt du foie. Or, comme dans le cas d'un déversement excessif de polypeptides (peptones mal digérées) dû à un apport exagéré de matériaux protidiques dans le régime, cette épuration ne se manifeste pas au pre- 
mier passage dans le foie. Il faut de multiples traversées pour la réaliser totalement ainsi qu'il résulte des travaux de $\mathbf{M}$. le professeur Fiessinger. "En présence d'une trop grande quantité de ces corps azotés mal transformés, écrit ce savant, il n'y a pas de foie, même normal, qui puisse résister. "

Cette défaillance du foie peut avoir, comme on le sait, des conséquences multiples : dermatoses, urticaires, accidents anaphylactiques, troubles circulatoires, aérogastrie, ete.

Le poumon. L'infection d'origine intestinale peut se localiser au poumon. On cite des cas où tantôt le colibacille, tantôt l'entérocoque furent reconnus responsables de congestions avec épanchements pleurétiques, de pneumonies, voire de crises d'asthme.

Les veines. Les migrations bactériennes d'origine intestinale ou autre, peuvent provoquer l'infection de la paroi des varices et déterminer des phlébites (à staphylocoques surtout).

Le systè me nerveux. "Certains états névropathiques ou psychopathiques, certains syndromes hypocondriaques avec idées délirantes et mélancoliques, peuvent être sous la dépendance directe de l'exotoxine neurotrope du colibacille. " (VINCENT.)

L'opinion exprimée par ce savant s'est trouvée confirmée par

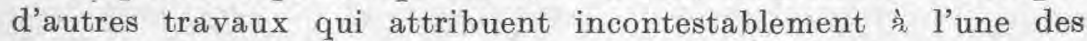
toxines colibacillaires certains troubles nerveux (fatigue, migraines, insomnies), et même mentaux (mélancoliee, irritabilité, découragement, etc.).

Cette action lointaine de l'auto-intoxication sur le système nerveux n'est d'ailleurs pas uniquement le fait des toxines microbiennes, mais aussi des poisons chimiques d'origine intestinale (phénols, indol, histidine, histamine).

Le rein. Si pour une cause quelconque, surcharge fonctionnelle ou autre, le rein voit sa perméabilité fléchir, les substances issues de la dégradation bactérienne de certains aminoacides restent dans le sang au lieu de s'éliminer dans les urines et provoquent des troubles souvent très graves d'intoxication généralisée (pouvant être par exemple un des facteurs principaux de l'urémie).

Au total donc, deux grands mécanismes paraissent se dégager de l'étude de ces intoxications :

$1^{0}$ La production intestinale de substances toxiques d'origine alimentaire ou microbienne ralentit ou compromet la neutralisation hépatique et l'évacuation rénale. Il en résulte l'intoxication générale de l'organisme avec ses nombreuses manifestations.

$2^{\circ}$ Le passage dans le sang et les humeurs du milieu intérieur 
des microorganismes eux-mêmes provoque des localisations entraînant des désordres nombreux et variés suivant la nature des agents, leur quantité ou leur virulence, le niveau de leur passage ou de leur fixation. Ces troubles ne sont pas des intoxications au sens biochimique du mot, mais autant de cas pathologiques dont l'étiologie réelle passe bien souvent inaperçue.

\section{$*^{*} *$ \\ DEUXIËIME PARTIE}

\section{PRINCIPES SCIENTIFIQUES DE LA THÉRAPIE LACTIQUE}

En remontant aux causes de ces troubles, groupés sous le terme général d'auto-intoxication ou de colibacillose, nous avons constaté que le facteur le plus important en est la composition bactériologique anormale du milieu intestinal.

Or, il n'est pas possible de supprimer radicalement les microorganismes variés qui sont les hôtes courants de l'intestin (germes de la putréfaction, colibacilles, entérocoque, staphylocoques, streptocoques) dont d'ailleurs, dans les états parfaitement normaux, la présence est sans inconvénient.

\section{Le problème à résoudre consiste simplement à empêcher ces germes de prendre ce développement et surtout d'acquérir cette virulence qui sont à l'origine des mécanismes que nous avons exposés.}

Le solution du problème ne peut être que la suivante : Créer et maintenir dans l'intestin un état physicochimique et bactériologique qui soit défavorable à ces germes dont nous voulons limiter ou même arrêter la prolifération et l'activité.

Quant au choix des moyens, l'état actuel de la technique bactériologique nous permettrait dans une certaine mesure de le fixer. Nous pensons qu'il convient tout d'abord d'interroger la nature ellemême pour y puiser s'il est possible les enseignements susceptibles d'orienter ce choix.

Le fait que l'intestin du tout jeune nourrisson ou du jeune animal est peuplé presque exclusivement de ferments lactiques - et de ferments lactiques spéciaux - constitue déjà une indication fondamentale.

L'observation, vieille comme le monde, suivant laquelle le lait cru abandonné à lui-même ne subit jamais la putréfaction, en est une autre qui confirme et peut-être explique la première.

Sur le plan technique de nos connaissances bactériologiques nous avons traduit ces faits en disant que la fermentation lactique s'oppose à la putréfaction - ou, d'une manière plus générale, que les ferments lactiques sont capables de s'opposer à la prolifération 
et à l'activité de nombreux autres microorganismes, en particulier de ceux qui constituent la flore intestinale de l'adulte. Or, c'est précisément là le but que nous poursuivons, le problème que nous avons à résoudre.

On s'explique ainsi qu'on ait depuis longtemps pensé à faire appel aux ferments lactiques pour maintenir la flore intestinale dans son état d'équilibre normal.

Sans entrer dans plus de détails - puisqu'aussi bien la cause est aujourd'hui entendue, du moins sur les principes - nous pouvons dono affirmer que l'un des moyens les plus certains de mener la lutte contre les hôtes indésirables du tube digestif jusqu'au point qui nous intéresse, c'est-à-dire entraver leur prolifération et inhiber leur activité, consiste à implanter dans l'intestin et à y maintenir une flore lactique, convenable et vigoureuse.

Le principe étant posé, il y a lieu de discuter les conditions de son application.

\section{Application des principes.}

Il faut reconnaître tout d'abord que la thérapeutique par les ferments lactiques, telle qu'elle a été comprise jusqu'à présent, a conduit à des échees retentissants. Certains, devant la disproportion constatée entre les résultats obtenus et les espoirs que cette technique avait fait naître, se sont découragés et ont perdu la foi dans la thérapie lactique. Mais ce ne sont pas les microorganismes eux-mêmes qu'il faut rendre responsables de ces échecs.

La plupart des préparations à base de ferments lactiques, en effet, ne correspondent en rien aux conditions strictes et sévères qu'elles devraient remplir. Plusieurs fabricants ont oru qu'il suffisait de faire pousser des ferments lactiques quelconques, dans un milieu non moins quelconque, et de les livrer dans un délai plus ou moins bien défini pour obtenir une implantation lactique véritable dans l'intestin. Et comme le contrôle de l'activité réelle de ces préparations ne peut être effectué ni par le médecin, ni par le consommateur, la raison véritable des échecs passe le plus souvent inaperçue.

II est désirable et possible de redresser cette situation. Mais pour y parvenir il faut tout d'abord dégager les conditions que la thérapie lactique doit remplir, c'est-à-dire établir les bases scientifiques sur lesquelles il convient de l'asseoir.

\section{Première condition : Choix de l'espèce microbienne}

Le terme de "ferments lactiques" embrasse un nombre considérable de microbes très variés n'ayant entre eux qu'un seul point commun, celui de transformer certains sucres en acide lactique. Mais des différences notables les séparent concernant leur optimum 
thermique, la rapidité et l'intensité de l'acidification, la nature et la quantité des produits accessoires formés, etc.

Or, tous les ferments lactiques ne sauraient convenir au but poursuivi ici. Une condition primordiale, en effet, doit dominer le choix de l'espèce à utiliser en thérapie lactique :

Puisqu'il s'agit de réaliser une implantation dans l'intestin, il est nécessaire que cette espèce puisse y vivre. Or, très rares sont les ferments lactiques capables non seulement de proliférer dans ce milieu très spécial, mais même d'y subsister. Seuls, les ferments lactiques d'origine intestinale comme ceux que l'on rencontre dans les fèces du très jeune enfant ou du très jeune animal, sont capables de s'adapter à l'intestin de l'homme, e'est-à-dire en somme de seréadapter à leur milieu originel.

Le Lactobacillus acidophilus de Moro, dont 1'habitat naturel est l'intestin des jeunes veaux, répond parfaitement à ces conditions. Mais il est le seul de tous les microbes du groupe des ferments: lactiques. Son optimum thermique très précis, tant pour son développement que pour son activité, est $37^{\circ}$, alors que pour d'autres dits thermophiles, l'optimum est $50^{\circ}$ et que, pour d'autres encore, utilisés dans l'industrie, il n'est que de $20-22^{\circ}$.

Sa résistance aux antiseptiques du milieu intestinal (bile par exemple) et à certaines substances toxiques produites au eours de la digestion (indol par exemple) est notablement plus grande que celle du Lactobacillus bulgaricus, ainsi qu'il résulte des travaux de KuLP récemment rappelés par D. BACH.

Ce dernier auteur qui fait autorité en la matière n'hésite d'ailleurs pas à écrire : «Le Lactobacillus acidophilus s'est révélé le seul ferment. lactique capable de s'implanter dans l'intestin de l'homme" (Presse Médicale, 11 août 1937).

Aux Etats-Unis la thérapie lactique est, à l'heure actuelle, basée exclusivement sur l'emploi de l'Acidophilus qui a conduit à des résultats très brillants. La plupart des échecs enregistrés en France dans ce domaine, viennent précisément de ce fait qu'on a utilisé des ferments quelconques sans se préoccuper de savoir s'ils pourraient s'implanter dans l'intestin.

Cette affirmation est-elle en contradiction avec les résultats souvent encourageants obtenus par l'usage répété du yaourt (Lactobac. bulgaricus) ? A vrai dire la consommation continue du yaourt aide incontestablement à réduire les putréfactions intestinales, mais: par un mécanisme tout différent de celui de l'acidophilus.

Le Bulgaricus dont l'optimum est de $50^{\circ}$ ne prolifère pas, ne: "s'installe" pas dans l'intestin. Il n'agit que par l'acidité qu'il a. produite dans le yaourt. Son rôle est limité au moment précis de son ingestion; il ne procure pas̀ cette bienfaisante concurrence 
vitale "germe à germe " qui résulte de l'emploi de l'acidophilus. Le yaourt, d'autre part, apporte, sous une forme généralement concentrée, les éléments du lait qui lui confèrent une grande valeur diététique et une certaine influence cholagogue.

La thérapie lactique par l'Acidophilus se propose un but différent qui est l'inhibition proprement dite des hôtes indésirables de l'intestin, et l'atteint d'ailleurs facilement, parfois même d'une manière définitive ainsi que cela a été vérifié.

L'extraordinaire vitalité de l'Acidophilus et sa haute résistance aux influences dysgénésiques supprime la nécessité des ingestions continues qui est de règle dans l'emploi du yaourt et permet, une fois l'implantation réalisée, de n'avoir recours qu'à des cures d'entretien espacées.

Quant aux autres ferments lactiques, l'espoir de le voir coloniser dans l'intestin doit êtrè maintenant complètement abandonné en parfaite connaissance de cause, même s'ils sont absorbés vivants et en grande abondance, ce qui n'est jamais le cas des préparations ayant plusieurs semaines d'âge.

En résumé, il est parfaitement connu que, seul, le Lactobacillus acidophilus peut s'adapter aux conditions spéciales du milieu intestinal et être utilisé avec chances de succès dans la thérapeutique par les ferments lactiques.

\section{Deuxième condition : Les ferments doivent être vivants et vigoureux}

Il est extrêmement difficile de préparer et de livrer au commerce des cultures de ferments lactiques dont tous les éléments soient encore vivants et virulents au moment de leur emploi par le consommateur. Certains auteurs ont cru pouvoir tourner la difficulté en prétendant que la thérapie lactique pouvait se faire à l'aide de germes morts, par analogie avec la théorie des bouillons vaccins.

Il y a là une double erreur :

10 Dans l'usage des bouillons vaccins on cherche, en général, à produire une immunité, c'est-à-dire une résistance à l'invasion du microbe dont on ingère la forme atténuée ou tuée.

Or, dans l'ingestion des ferments lactiques il n'est pas question de les voir pénétrer dans le milieu intérieur. Et si tel était le cas, c'est tout le contraire d'une immunité que l'on rechercherait, à l'inverse des germes contre lesquels on veut se défendre.

$2^{\circ}$ La théorie du pigment jaune de WARBURG (ou flavine) que ces auteurs invoquent également, ne saurait justifier l'emploi de ferments morts. Certes, les ferments lactiques sont en général très riches en flavine (qui n'est autre que la vitamine B2) puisqu'ils en contiennent plus de 100 milligrammes par kilogramme, contre 
30 à la levure de bière considérée jusqu'à ces dernières années comme la source la plus riche de cette vitamine. Les ferments lactiques tués par la chaleur contiennent la même dose de cette précieuse flavine. D'où l'idée d'en faire une source commode de facteur B2 utile à la croissance des ferments lactiques déjà présents (?) dans l'intestin.

Mais le résultat ne peut qu'être incontestablement meilleur si les microorganismes que cette vitamine doit favoriser sont nombreux et vivants. Le facteur B2 ne profitera certes pas aux cadavres microbiens!

D'ailleurs, il est sage croyons-nous d'adopter l'opinion si opportunément exprimée par M. BACH sur ce sujet : l'influence du ferment de WARBURG ne doit pas être invoquée pour expliquer la bienfaisance des ferments lactiques, puisqu'aussi bien certains des germes contre lesquels nous voulons lutter en contiennent encore plus : les bactéries butyriques par exemple en contiennent 136 milligrammes par kilogramme (WARBURG, Bioch. Zeitsch, , 266, 377, 1933). La vitamine B2 ne devrait-elle pas exalter aussi ees microorganismes indésirables au même titre et plus peut-être que les ferments lactiques?

En fait, une étude objective des principes de la thérapie lactique nous montre à l'évidence que les ferments doivent être absorbés vivants et même en état de pleine virulence puisqu'ils doivent à la fois se fixer dans des conditions souvent difficiles et concurrencer efficacement des germes déjà installés et vigoureux. Ils doivent s'aequitter de ce double rôle par l'influence de la fermentation lactique qu'ils établissent "in situ » et maintiennent sans arrêt, et par le rôle inhibiteur d'ailleurs mal connu dont ils sont eapables par leur seule présence, même en milieu parfaitement neutre.

Ce mécanisme est évidemment conditionné par une prolifération intense et par une grande activité. Cela suppose done bien que les ferments soient vivants, restent vivants et pas seulement de cette vie minimum qui permettrait à un repiquage en milieu eugénésique de donner une culture positive, mais de cette vitalité florissante qui conditionne l'épanouissement de la pullulation dans un milieu naturellement dysgénésique.

Sur ce point particulier de la virulence des souches de Lactobacillus il y a lieu de se montrer extrêmement exigeant et on en comprend les raisons.

\section{Troisième condition : Les ferments doivent être absorbés en grande abondance}

Cette condition est évidemment moins importante que les deux précédentes. Il serait sans intérêt, en effet, d'absorber de très 
grandes masses de germes s'ils sont incapables de vivre dans l'intestin et à plus forte raison s'ils sont morts avant cette ingestion.

Mais en supposant que l'espèce ait été bien choisie (Lactobac. acidophilus) et que les éléments en soient vivants et parfaitement virulents, il est encore nécessaire qu'ils soient nombreux.

Lorsqu'on suit la courbe de croissance d'une culture microbienne quelconque, on s'aperçoit que la phase d'accroissement proprement dite est précédée d'une phase d'adaptation au cours de laquelle la multiplication est pratiquement nulle. Cette phase initiale dure un temps variable, parfois très court, parfois de quelques heures ou davantage suivant les conditions physiques, chimiques de la culture, suivant l'état physiologique antérieur du microbe, etc.

Dès que la croissance commence, elle s'oriente presque immédiatement vers une allure très caractéristique, franchement logarithmique. L'expression mathématique de la courbe de croissance traduit alors elairement qu'il y a intérêt à disposer au départ du plus grand nombre possible d'individus. En effet :

La plupart des microbes, les bacilles lactiques en particulier, se multiplient par scissiparité. Chaque individu en donne deux; ces deux nouveaux germes en donnent quatre, etc. Le nombre des microorganismes présents croît comme les termes d'une progression géométrique de raison deux.

Soient $n$ le nombre de germes au début de la phase logarithmique de croissance, $N$ le nombre de bactéries présentes au temps $t, x$ le nombre de générations produites pendant ce temps $t$.

On a évidemment : $N=n \cdot 2^{x}$.

C'est la formule classique donnant la valeur d'un terme quelconque d'une progression géométrique : $l=a q^{\text {n.1 }}$ où $x$ (nombre de générations) $=n-1$ (nombre de termes précédant le terme $l$ )

Cette fonction exprime, outre le caractère exponentiel prévu de la croissance, le fait qu'à un temps donné, le nombre de germes présents est proportionnel au nombre de germes à l'origine.

A tout moment l'état quantitatif de la culture est conditionné étroitement par son état initial. Il en est le reflet exact.

Si maintenant on met en parallèle deux cultures de richesse initiale différente, elles présenteront à tout instant un décalage qui ne sera jamais comblé pendant la phase logarithmique de croissance, e'est-à-dire celle pendant laquelle la vitesse de multiplication est constante. Le nombre de germes au temps $t$ de la culture la plus riche, ne sera atteint par la culture la plus pauvre qu'au temps $t^{\prime}$ nettement différent de $t$.

Le calcul montrerait encore que ce décalage de temps est fonction de la différence des richesses à l'origine. Cè qui revient à dire que plus une culture est pauvre au départ, plus l'obtention d'un 
effet biologique donné est retardée. Or, si ce retard peut être sans importance "in vitro" dans un milieu eugénésique, il n'en est pas de même dans le milieu intestinal. Ici, retarder l'épanouissement de la culture par des ingestions trop pauvres, c'est risquer de le compromettre ou tout au moins de l'entraver.

Il est done nécessaire d'agir par des apports massifs de microorganismes, seuls capables de réaliser cette concurrence vitale efficace qui est à la base de la réussite de l'implantation lactique.

$$
*^{*} *
$$

En résumé, une étude critique sommaire de la thérapie lactique nous montre qu'il est indispensable de remplir les conditions suivantes :

$1^{0} \mathrm{Ne}$ faire appel qu'à un ferment d'origine intestinale, seul capable de se fixer et de se développer dans l'intestin.

$2^{\circ}$ N'utiliser que des germes vivants et en pleine virulence.

$3^{\circ} \mathrm{N}^{\prime}$ employer que des cultures très riches.

\title{
LES MAMOCOQUES EN FROIMAGERIE
}

\author{
par \\ Costantino GORINI (Milan)
}

Notre distingué collègue, W. Dorner, écrit dans "Le Lait», de mai dernier, page 451, ce qui suit : "Les microcoques de la mamelle sont, dans la règle, inoffensifs. Certains auteurs (GORINI) estiment qu'ils jouent un rôle favorable dans la maturation du fromage. Pour le Gruyère et l'Emmental, rien ne laisse supposer qu'il en soit ainsi. "

Nous le remercions parce qu'il nous donne l'occasion de répéter ici ce que nous avons dit déjà maintes foìs, et même récemment dans le Volume Jubilaire de Robert BurRI (1937), et au Congrès mondial de Laiterie de Berlin (1937). Le rôle fromager des mamocoques, comme celui de tous les acidoprotéolytes, est complexe ; mais avant tout, par leur action solubilisante sur la caséine, ils sont des activateurs des ferments, lactiques, qui s'avantagent plutôt des produits caséolytiques. Or, pour ne pas accepter que les mamocoques jouent un rôle favorable dans la maturation du Gruyère et de l'Emmental, il ne sufíit pas de contester leur présence dans le fromage, il faut démontrer qu'ils ne se sont point développés dans le lait avant son arrivée à la laiterie, dans les cuvettes de repos et dans la chaudière même. A ce propos, nous rappellerons les belles recherches de Hanusch (Wien. Milchw. Ber., Dezember 1933) qui a trouvé cons- 